\title{
Interference analysis and operability envelopes design for deepwater parallel strings
}

\author{
Jingyu Zhu, Kang Liu*, Guoming Chen, and Gaogeng Zhu \\ Centre for Offshore Engineering and Safety Technology, China University of Petroleum (East China), Qingdao, China
}

Received: 12 December 2018 / Accepted: 14 May 2019

\begin{abstract}
Dual derrick operations are widely used for field development during offshore drilling. During this process, complex operations and complicated hydrodynamic interaction may contribute to the interference collisions of parallel strings. In this context, this study addresses interference collisions for parallel strings during the deepwater dual derrick operation. To analyze the response of parallel strings a mechanical model for deepwater parallel strings is established. Moreover, Huse wake model and strip model are used for calculating the hydrodynamic influence in different wake fields. The research results validate that the collision will occur during the operation considering the hydrodynamic wake shielding effects and interference evaluation criterion. The increasing platform offsets and surface current are the leading causes of parallel strings collisions. To avoid the risk of interference collisions an innovative procedure for operability envelopes is developed by synthesizing the platform offsets and surface currents. The proposed operability envelopes method for parallel strings is automatically completed which can save much workforce and resources. A case study on deepwater drilling in the South China Sea has been applied to verify the effectiveness of these methods. Besides, the proposed methodology can effectively reduce collision accidents and provide technical support for the offshore oil and gas exploration.
\end{abstract}

\section{Introduction}

Reducing the cost and improving the efficiency of deepwater drilling operations have become a focus for the researchers and field workers. To address that, a dual derrick drilling platform was developed. Compared to traditional drilling platform, the efficiency of dual-activity drilling with up to $20 \%-40 \%$ time saving on deepwater oil development. However, many researchers indicate that the interference between adjacent risers is a significant concerned issue (Huse, 1993, 1996; Rustad et al., 2009). A collision probability of parallel strings also exists during the simultaneous operation of the upstream riser and downstream strings. Although the impact may not lead to failure directly the impact of risers is believed to be the fundamental cause of cracks and induce fatigue failure in the long term ( $\mathrm{He}$ and Low, 2012). Due to the heavier Blowout Preventer (BOP) of the upstream riser, the clashing may lead to a more severe consequence for dual derrick operations and result in a downstream string fail. Several collision events occurred during the process of lowering the BOP of the riser in deep water operation (Drumond et al., 2018). As the offshore

* Corresponding author: 1kzsww@163.com industry moves to deeper waters, the interference collision of parallel strings is becoming a more and more important issue with their increased lengths. Therefore, a general approach to reducing the risks of clashing for parallel strings system is desirable.

The parallel strings would subject complex hydrodynamic forces when ocean current flows around them. Even though the literature on the parallel strings collisions have little been reported for dual derrick operation, much research has been done on the riser interferences (DNV-RP-F203, 2009; Huse, 1993, 1996, Rustad et al., 2009). According to the previous experimental and numerical research, the Vortex-Induced Vibration (VIV), WakeInduced Oscillation (WIO) and wake shielding effects are leading causes for riser interferences (DNV-RP-F203, 2009; Fontaine et al., 2013; Huang, 2010; Sumner, 2010; Wu et al., 2003). Sagatun et al. (2002) presented a simulator to assess the collision probability for the adjacent risers closing to each other, considering the hydrodynamic interaction of WIO and VIV. The VIV effects of the upstream riser can increase the effective drag forces when the downstream riser locate in the wake of the upstream riser experiences reduced loading (Nygård et al., 2001). Kalleklev et al. (2003) indicated that the hydrodynamic interaction between two neighboring risers mainly influences 
downstream riser rather than the upstream riser. Furthermore, the dynamic response of vortex and wake-induced vibrations of tandem cylinders is investigated under near and far wake interferences respectively (Huera-Huarte and Bearman, 2011; Huera-Huarte and Gharib, 2011). Zhang et al. (2018) proposed a time domain prediction method from experimental data for VIV of flexible risers. Yuan et al. (2018) proposed an alternative time domain forcedecomposition model for flexible risers to predict VIV response under both steady and oscillatory flows. Regarding WIO, Blevins wake field model can be used to simulate and account for the lift forces (Blevins, 1994). The model and interaction are still not fully understood and advocated in the riser clearance assessment (DNV-RP-F203, 2009; He and Low, 2014).

Thus, some safety measures have been proposed to prevent and avoid impact between neighboring risers. Rustad et al. (2008) provide a PI-controller to avert the clashing for the top tensioned risers. An approach is presented to determine the maximizing installation envelopes for drilling riser under the criterion of "no clashing principle" (Campbell et al., 2013). A constructive design of boundary controllers is proposed to stabilize the transverse motion of flexible marine risers (Do, 2018). Connected operability envelopes of the deepwater drilling riser are determined considering the vessel offset and surface current (Ju et al., 2012). Wang et al. (2015) developed a safety operation window for riser in installation through analyzing three kinds of mechanical behavior. Liu et al. (2019) adopted a self-design procedure to determine the operability envelope for marine production string self-induced vibration based on pipe-in-pipe mode. To evaluate the collision probabilities accurately, the collision probability for two flexible risers is determined by taking into account the hydrodynamic wake interference, environment loads, and surface floater motions (Fu et al., 2017, 2018). Considerable efforts have been devoted to the study of riser interferences, leading to further investigations of parallel strings.

As the exploration of offshore oil resources moves into deepwater or ultra-deepwater, risers are becoming increasingly slender and the clashing probability is increasing in turn. Thus, the anti-collision for the whole parallel strings need to be studied during deepwater dual derrick operation. This paper proposes an innovative procedure of operability envelopes to avoid clashing between parallel strings based on an established mechanical model for parallel strings by taking the wake shielding effects into account. Besides, the proposed method is efficient and user-friendly and operability envelopes are automatically completed, which can save much workforce and resources. In addition, safe areas can be provided in the operability envelopes considering the platform offset and surface current synthetically during the lowering process of the upstream riser/BOP for the dual derrick drilling operation. The rest of the paper is organized as follows. Section 2 provides a background and theory of foundations for parallel strings. Section 3 introduces the proposed methodology. Section 4 illustrates the research results by applying the proposed methodology in the South China Sea. Finally, Section 5 summarizes the main conclusions.

\section{Preliminaries}

\subsection{Background}

The dual activity drilling process is designed to reduce critical path activity and improve efficiency in both exploration and development drilling applied in deepwater drilling environment (Wilburn et al., 1998). The concept of a dual-activity platform has been in existence since 1995 which was constructed by Transocean Offshore Inc. in February 2000 (Munch-Søegaard and Nergaard, 2001). The essence of dual derrick drilling is to equip the derrick on the platform with two drilling rigs. One significant advantage identified is that the auxiliary rig is out of the critical path while drilling progresses with the main rig (Hall et al., 1999). Figure 1 shows one rig conducts all top holes operations, drilling, tripping, running pipe while the other is running the BOP and riser (Liu et al., 2017; Munch-Søegaard and Nergaard, 2001).

The dual activity drilling process means that the main rig is in a $\mathrm{BOP} /$ riser drilling mode. Meanwhile, the auxiliary rig drills the top hole and install surface casings in the next well (Hall et al., 1999). The detailed procedure of the dual derrick drilling operation includes the following seven steps (Dong et al., 2011; Yue et al., 2009): (a) Drilling ship takes its place and completes the pre-drill preparation, the main rig lowers drilling template and fixes it to the seabed. (b) The main rig performs the jetting conductor operation, the auxiliary drilling rig assembles the drilling string. (c) The installation of surface casing is completed by the main rig, the casing head is placed on the conductor head. At the same time, the auxiliary drilling rig connects and lowers the BOP and riser in turn. (d) The platform is displaced after the running operation, after which the auxiliary rig is moved above the wellhead, the riser group and the BOP are installed. (e) The auxiliary rig drills and the intermediate casing is run and cemented by the main rig. (f) The primary and auxiliary rigs are drilling in parallel,

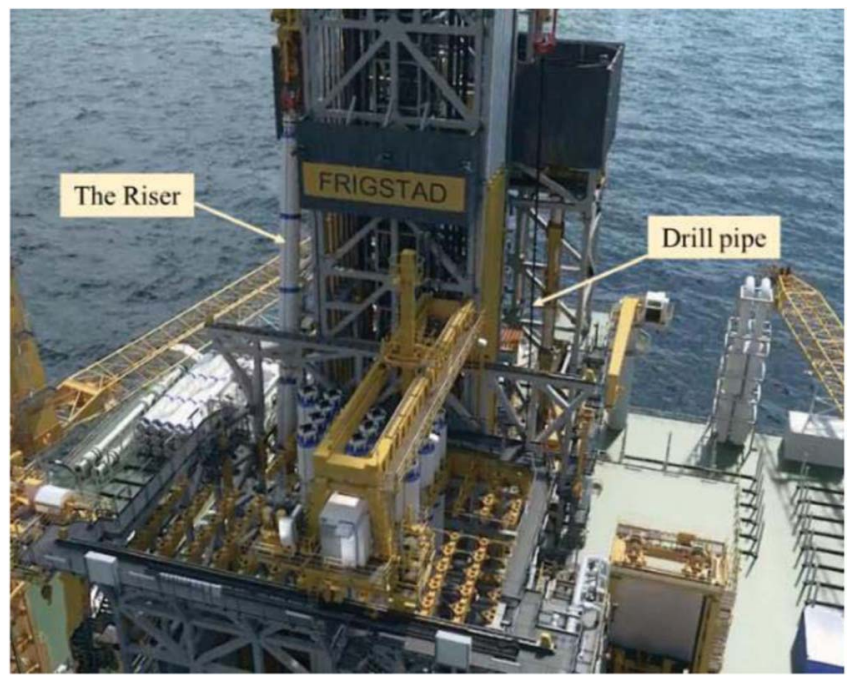

Fig. 1. Parallel strings working for the deepwater dual derrick platform. 
and then the primary rig drills to the reservoir and lowers capital string through the riser. Meanwhile, the X-trees are fitted by auxiliary rig. (g) Finally, the completion and testing are conducted.

Dual rigs can significant improve the efficiency of drilling operations compared with a single rig operation, but it will encounter a severe problem about interference collision of parallel strings. The space between strings is limited at the topside onboard the platform as well as the distance between dual derrick (Rustad et al., 2009). In practice, strings impacts are not allowed under normal or even extreme conditions (He and Low, 2014). The BOP stack is connected to the lower end of riser once a collision occurs, which will cause severe damage to the drill string. The interference probability of parallel strings is increasing with their increased riser lengths. The operation diagram of parallel strings for the dual derrick platform is shown as Figure 2. However, the mechanism of strings interference is very complex and whether adjacent strings will collide or not depending on many factors. The most important influencing factors include loading environment, strings space, shielding effects, VIV effects (DNV-RP-F203, 2009). In practice, the platform is forbidden to rotate before the "second section" that is, one stage before the intermediate casing is installed during the drilling operation. Otherwise, the platform can prevent the parallel strings and inflow current from being in the same plane by rotating a certain angle, thus avoiding the interference collision of the parallel strings. Hence, interference analysis between adjacent

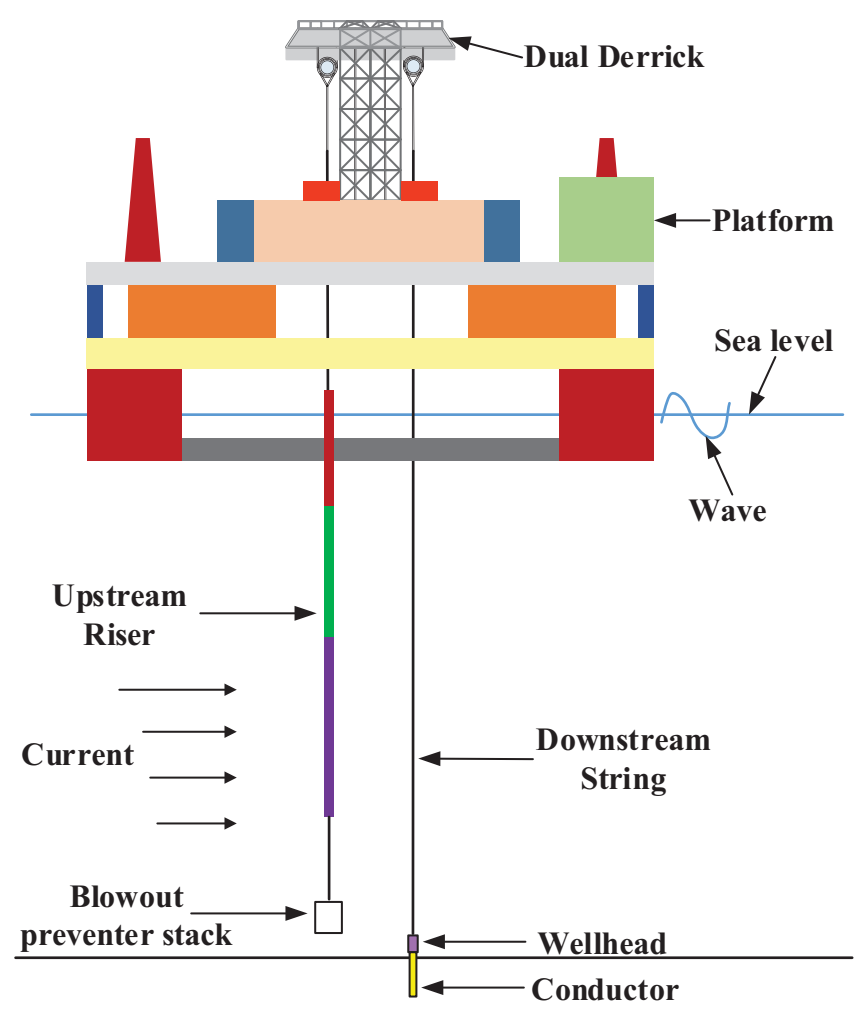

Fig. 2. Operation diagram of parallel strings for the dual derrick platform rig. strings is highly significant research during the running operation of the upstream riser for dual derrick platform.

\subsection{Mathematical model}

The deformation differential equation of marine strings is established before further studies. The marine strings are assumed vertical at the initial position firstly. The coordinate system is as follows: where $z$ is the vertical height, $\mathrm{m} ; y$ is the horizontal displacement, $\mathrm{m}$ (Chang et al., 2017; Ju et al., 2012). The differential equation is:

$$
\begin{gathered}
\frac{\mathrm{d}^{2}}{\mathrm{~d} z^{2}}\left[\mathrm{EI}(z) \frac{\mathrm{d}^{2} y}{\mathrm{~d} z^{2}}\right]-T(z) \frac{\mathrm{d}^{2} y}{\mathrm{~d} z^{2}}-W(z) \frac{\mathrm{d} y}{\mathrm{~d} z}=f(z), \\
T(z)=T(0)+\int_{0}^{z} M(z) \mathrm{d} z \\
M(z)=\frac{\pi}{4}\left(D^{2}-D_{i}^{2}\right) \rho_{s}+\frac{\pi}{4} D_{i}^{2} \rho_{m}+\frac{\pi}{4} D^{2} \rho_{w}\left(C_{w}-1\right), \\
f(z)=\frac{D}{2} \rho_{w} C_{D}\left(v_{w}+v_{c}\right)\left|v_{w}+v_{c}\right|+\frac{\pi D^{2}}{4} \rho_{w} a_{w} \\
+\frac{\pi D^{2}}{4} \rho_{w} C_{w} a_{w} .
\end{gathered}
$$

In the equation, $M$ is the string mass unit length, $\mathrm{kg} / \mathrm{m} ; E$ is the elastic modulus, $\mathrm{Pa}$; $I$ is the moment of inertia, $\mathrm{m}^{4} ; T$ is the axial tension of strings, $\mathrm{N} ; T(0)$ is the top tension of the string, $\mathrm{N} ; D$ is the equivalent inertial force diameter of strings, $\mathrm{m} ; D_{i}$ is the internal diameter of strings, $\mathrm{m} ; s$ is the density of riser material, $\mathrm{kg} / \mathrm{m}^{3} ; m$ is the density of the liquid in the strings, $\mathrm{kg} / \mathrm{m}^{3} ; w$ is the density of sea water, $\mathrm{kg} / \mathrm{m}^{3} ; C_{M}$ is the inertia force coefficient, dimensionless; $f$ is the combined force of wave-current acting on the unit length of the strings, $\mathrm{N} / \mathrm{m} ; C_{D}$ is the drag coefficient, dimensionless; $v_{w}$ is water particle velocity caused by waves, $\mathrm{m} / \mathrm{s} ; v_{c}$ is water particle velocity caused by currents, $\mathrm{m} / \mathrm{s} ; a_{w}$ is water particle acceleration along the radial direction of the string caused by waves, $\mathrm{m} / \mathrm{s}^{2}$.

How to calculate the inflow velocity on the downstream string is the critical issue in these mathematical equations. Due to the presence of fluid, the hydrodynamic interaction which occurs between two adjacent strings can affect the inflow velocity on the string. Much theoretical research and numerous experiments are performed to investigate hydrodynamic interaction in steady current (Huse and Kleiven, 2000; Blevins, 1994; Zdravkovich, 2003). A semiempirical wake field model in the steady flow field was proposed by Huse to account for the hydrodynamic interaction between the two strings when analyzing the inflow velocity of the downstream string in Figure 3 (Huse, 1993, 1996).

The hydrodynamic loading on the downstream string will be influenced by the wake field generated by upstream string (Kalleklev et al., 2003). In the equilibrium state, the resulting inflow on downstream riser considering shielding 


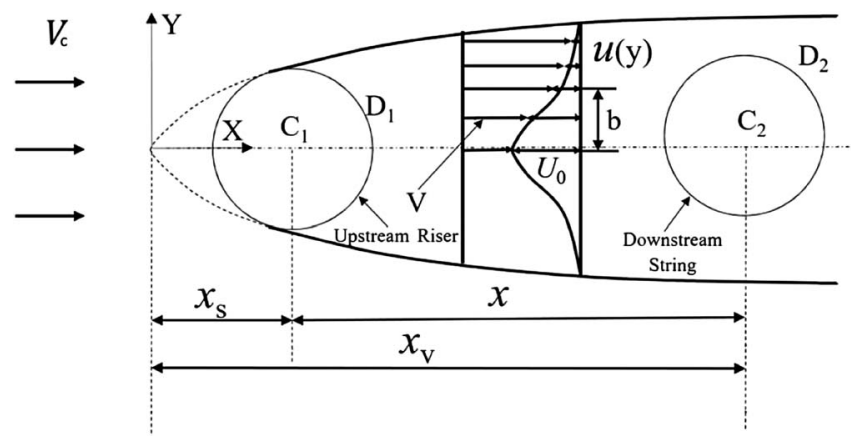

Fig. 3. Huse semi-empirical wake model of strings in stationary flow field (DNV-RP-F203, 2009).

effects from the upstream riser is determined by the Huse semi-empirical wake model and the iterative method of the wake velocity specified according to DNV-RP-F203 (2009). The inflow velocity in the wake field can be computed using the following expressions:

$$
\begin{gathered}
x_{v}=x+x_{s}=x+4 D_{1} / C_{d 1}, \\
b=k_{1} \sqrt{C_{d 1} D_{1} x_{v}}, \\
V=V_{c}-u=V_{c}-k_{2} U_{0} \sqrt{\frac{C_{d 1} D_{1}}{x_{v}}} e^{-0.639\left(\frac{y}{b}\right)^{2}},
\end{gathered}
$$

where, $x_{\mathrm{s}}$ is virtual source distance from upstream string $C_{1}$ to virtual, $\mathrm{m} ; x_{\mathrm{v}}$ is a distance from downstream string $C_{2}$ to virtual, $\mathrm{m} ; x$ is a distance from upstream string to downstream string, $\mathrm{m} ; D_{1}$ is the diameter of the upstream string, $\mathrm{m} ; C_{\mathrm{d} 1}$ is the reference drag force coefficient of upstream string; $V_{\mathrm{c}}$ is the inflow velocity on the upstream string, $\mathrm{m} / \mathrm{s} ; u$ is the reduced velocity in the wake of upstream string, $\mathrm{m} / \mathrm{s} ; U_{0}$ is the maximum reduced velocity in the wake of upstream string, $\mathrm{m} / \mathrm{s} ; V$ is the resulting inflow on a downstream riser, $\mathrm{m} / \mathrm{s} ; y$ is the distance away from the center line of incoming velocity profile; $k_{1}=0.25$ and $k_{2}=1.0$ are empirical constants for a smooth cylinder; $b$ is half-width profile of inflow velocity on the downstream string, $\mathrm{m}$.

\section{Methodology}

\subsection{Developed model for parallel strings}

Assessment of hydrodynamic interaction is a significant issue for string interference evaluations. Therefore, it is necessary to establish a mechanical model of parallel strings firstly. When the parallel strings work together, the upper end of the riser and drilling string are connected to the drilling operation platform (Hall et al., 1999). Nevertheless, the lower end of the riser is suspended by the BOP, and the string is connected to the subsea wellhead. The working area of dual derrick platform is generally in the deep waters. Owing to the shielding analysis between strings, when the platform is offset, the simultaneous action of the current and the waves are prone to cause large transverse deformation of the strings, resulting in collision of the parallel strings. It is assumed that the direction of the parallel arrangement strings is consistent with the current, and the analysis model of parallel strings is shown in Figure 4.

The mechanics model of parallel strings system is an ordinary differential equation for the deformation of beams in a vertical plane under lateral loads. An impact of the crash will emerge between the strings when the parallel string collides. A developed formula for calculating the hydrodynamic force and impact force of parallel strings is presented as

$$
\begin{aligned}
& \frac{\partial^{2}}{\partial z_{i}^{2}}\left(\mathrm{EI} \frac{\partial^{2} y}{\partial z_{i}^{2}}\right)-\frac{\partial}{\partial z_{i}}\left(T(z) \frac{\partial y}{\partial z_{i}}\right)+M(z) \frac{\partial^{2} y}{\partial t^{2}} \\
& \quad=f\left(z_{i}, t\right)+F_{i},
\end{aligned}
$$

where $F$ is the impact force unit length between strings, $\mathrm{N} / \mathrm{m} ; i(i=1,2)$ indicates different strings.

Furthermore, the revised Morrison equation is needed to determine the hydrodynamic load $f(z, t)$ considering the effect of the relative motion of the riser (Liu et al., 2013):

$$
\begin{aligned}
f(z, t)= & \frac{\pi D^{2}}{4} \rho_{w} a_{w}+\frac{\pi D^{2}}{4} \rho_{w}\left(C_{M}-1\right)\left(a_{w}-\frac{\partial^{2} y}{\partial t^{2}}\right) \\
& +\frac{D}{2} \rho_{w} C_{D}\left(v_{w}+v_{c}-\frac{\partial y}{\partial t}\right)\left|v_{w}+v_{c}-\frac{\partial y}{\partial t}\right|,
\end{aligned}
$$

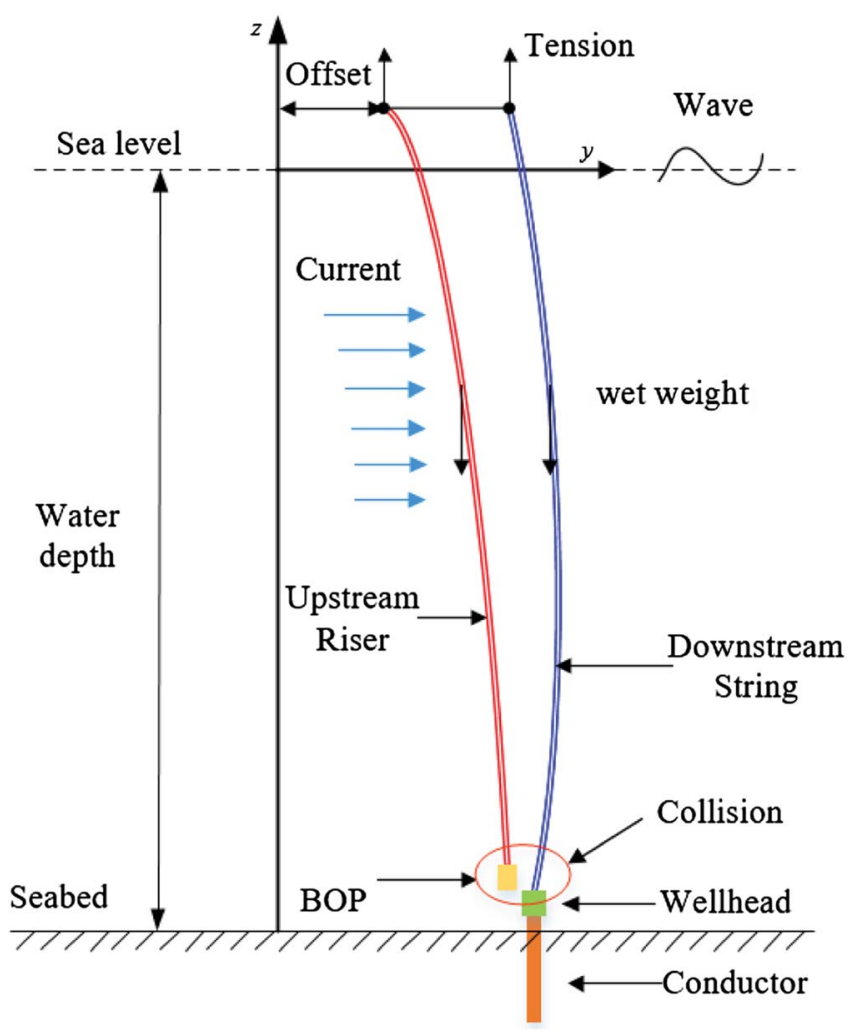

Fig. 4. Mechanics model of parallel strings system. 
where, $\partial^{2} y / \partial^{2} t$ is string particle acceleration, $\mathrm{m} / \mathrm{s}^{2} ; \partial y / \partial t$ is string particle velocity respectively, $\mathrm{m} / \mathrm{s}$.

The aforementioned Huse wake model is only suitable for calculating the hydrodynamic influence on the downstream flow field in a $2 \mathrm{D}$ plane. The hydrodynamic interaction between parallel strings in a 3D space can be analyzed utilizing a "strip model" (Rustad et al., 2009). Different wake fields can be applied in different slice layers due to the different currents at different water depths in Figure 5. Herein, the parallel strings system is divided into many strips along the depth of the water, and each strip is equivalent to the upstream and downstream strings with uniformly-spaced. In the vertical direction, the hydrodynamic interaction force is computed using a pre-established two-dimensional wake model in each strip (Rustad et al., 2009; Zdravkovich, 1997). In each strip, the interaction force on the downstream string depends on the relative distance between the two strings and the relative velocity between the fluid and the string. Thus, the application of strip theory in the mechanical model makes the computing results more accurately.

\subsection{Developed framework}

As mentioned in the previous section, we propose an efficient and user-friendly method to prevent the parallel strings collisions, which combines python language and finite element analysis. The overall framework of the proposed model is shown in Figure 6. Operability envelopes are designed to answer whether the drilling operation can be carried out and how much risk the operation activity has for a given environmental condition. The procedure is implemented by python language and finite element model to determine the operability envelopes by synthesizing the

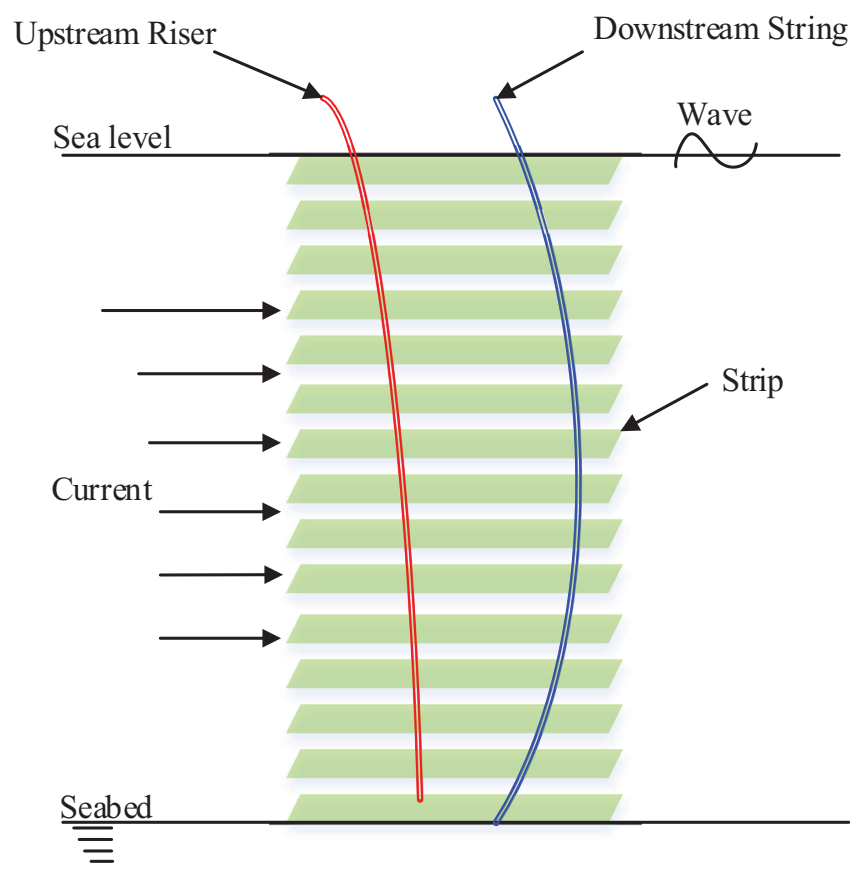

Fig. 5. Strip theory of interference analysis.

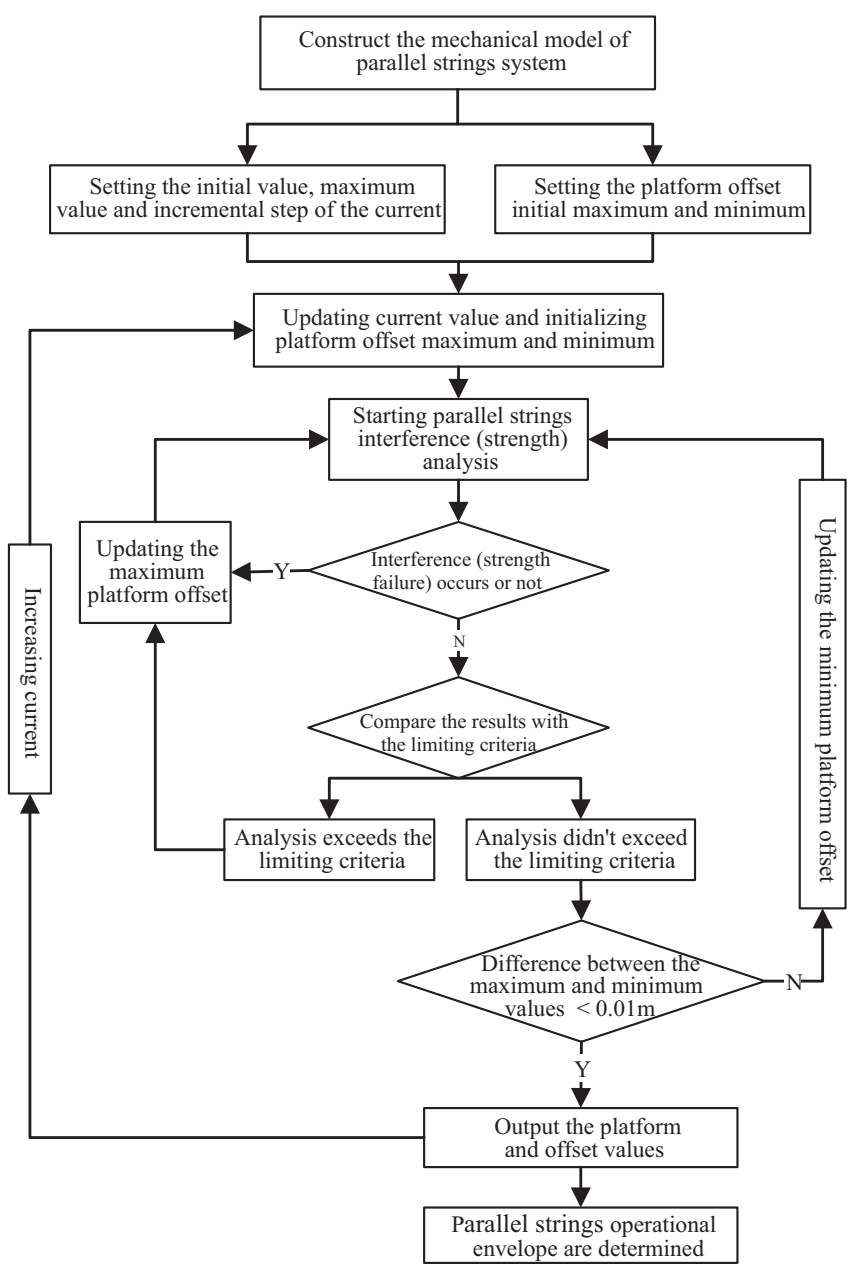

Fig. 6. The framework of operability envelopes for parallel strings.

critical platform offset and surface current efficiently under the serial cylinder operation conditions. Besides, the envelope is automatically completed which can save much workforce and resources.

The framework of operability envelopes consists of several parts: mechanical model building, iterative analysis, interference judging, and envelopes determined. In the first part, the finite element model of the parallel strings system is constructed firstly, which considers the effect of hydrodynamic interaction and strip theory adequately between parallel strings.

In the second part, the iterative analysis of parallel string system is carried out, taking the surface current velocity as the external loop and the platform offset as the internal recycling. Specifically, the iteration procedure can be divided into the following six steps:

Step 1: Determine the current velocity on the downstream string according to the initial spacing and the diameter of the strings and divide the two strings into some strips based strip theory.

Step 2: Set the initial value, maximum value and incremental step of the current and platform offset and limiting criteria. The detailed explanation for interference 
evaluation criterion and strength criteria of parallel strings is presented in Section 3.3.

Step 3: Start parallel strings interference/strength calculation.

Step 4a: Compare the calculation results with the interference evaluation criterion, if the spacing between two strings is smaller than the judging criterion or the contact force is generated between two strings, which can be judged that the two strings have interfered and the calculation is completed. If not, go to the next step.

Step 4b: Compare the calculation results with the strength criteria, if the equivalent stress, bending moment, deflection angle are greater than the criteria, which can be judged that the parallel strings may failure and the calculation is completed. If not, go to the next step.

Step 5: The resulting inflow on the downstream string is recalculated according to the Huse wake model. Moreover, the spacing, drag coefficient or current loading at different positions on the downstream string is updated according to the new result. Repeat steps 4 until convergence is achieved.

Step 6: Ultimately, interference (strength failure) occurs or not between the two strings in the steady state can be judged according to the limiting criteria, and the calculation is completed.

Interference judging and comparison results are carried out in the third part, if the parallel strings interference, output the value of the platform offset and surface current. Otherwise, the program continues normally. Meanwhile, compare the results of equivalent stress, bending moment and angle with the criteria of operability envelopes and output the value of the platform offset and surface current if results exceed the strength criteria.

Finally, a series of critical values for platform offset under different surface current are obtained using a nonlinear search algorithm to calculate in the fourth part. Then, the operability envelopes for parallel strings are determined to take the intersection of safety operation scope and interference boundary for parallel strings.

\subsection{Limiting criteria of parallel strings}

The limiting criteria of parallel strings include two parts the interference evaluation criterion and the strength criteria. The former criteria aim to avoid interference collision of the parallel strings. Shielding effects and VIV effects are the most critical effects of relevance for the evaluation of string interference. The presence of wake shielding effects, which results in a loss of current velocity on the downstream string, can thereby reduce the current forces on the downstream string and tending to bring the strings closer. Another factor is VIV effects, which may cause a string deviation about a magnitude of one diameter for each string (DNV-RP-F203, 2009). A minimum clearance criterion was adopted to avoid collision of the strings considering the shielding effects and VIV effects of upstream and downstream strings (DNV-RP-F203, 2009). Therefore, the minimum clearance between the strings must satisfy the requirement: $\Delta \geq D_{1}+D_{2}$ in case of strings collision without considering any safety factor. $D_{1}$ and $D_{2}$ are the outer diameters of the upstream and downstream strings respectively; $d$ represents the distance between the centers of two strings; $\Delta$ indicates the clearance between two strings. Moreover, it can also provide a reference for interference collision of parallel strings for dual derrick operation.

The latter criteria correspond to ensure structural strength of parallel strings. Safety drilling operations of parallel strings are mainly limited by equivalent stress, bending moments, the angle of deflection, and interference between strings. The restrictive criteria of operability envelopes for parallel strings are presented in Table 1. Among them, the restrictive conditions of equivalent stress for the riser and the strings, and the maximum bending moment of wellhead are designed to ensure the structural integrity of the string system during the drilling operation. Furthermore, the maximum deflection angle of the wellhead aims to prevent the abrasion of the wellhead during the drilling operation, and ensure that the drill pipe goes straight through the wellhead without incident. Besides, the "no collision allowed" design principle is cited to prevent collision occurrences of parallel strings (He and Low, 2012).

\section{Case study}

\subsection{Configuration and simulation for the parallel strings system}

A case study for seventh generation deepwater drilling platform of the dual derrick in the South China Sea is conducted to illustrate this methodology, and the operating water depth is $1300 \mathrm{~m}$. Referencing the "The Blue Whale 1" deepwater dual derrick platform, the distance between the upstream riser and downstream string is $16 \mathrm{~m}$. Concretely, 1-year return period of sea condition parameters is adopted in this study. The drag coefficient is 1.2 in the water depth of $0 \sim 150 \mathrm{~m}$ and 0.7 at depth over $150 \mathrm{~m}$. The inertia force coefficient is 2.0. The specific parameters of the parallel strings system are shown in Table 2 .

The finite element model of the parallel strings system has been established by finite element software, and the drill pipe, as well as the riser, were simulated based on the basic parameters and proposed theory model in the previous Section 3. The casual contact between the upstream riser and the downstream drill pipe was simulated by contact element and flexible joint with ball joint unit. Vertical tension is applied to the top of riser and drill pipe to simulate the tension of the tensioner and hook. Finally, current, temperature, and pressure load and top offset were applied and simulated by the hydrodynamic analysis model. Furthermore, the analysis procedure of operability envelopes for parallel strings is implemented with python language based on the mechanical model.

\subsection{Interference analysis of parallel strings}

\subsubsection{Results of interference analysis}

The interference analysis of parallel strings is investigated in this section. The surface current is $0.7 \mathrm{~m} / \mathrm{s}$, and the results of the displacement and rotation are shown in 
Table 1. Strength criteria of operability envelopes for parallel strings (DNV-OS-F201, 2010).

\begin{tabular}{lc}
\hline Limiting factors & Restrictive conditions \\
\hline The maximum equivalent stress of the riser/yield strength & 0.80 \\
The maximum equivalent stress of the string/yield strength & 0.67 \\
Maximum bending moment of wellhead/yield strength & 0.80 \\
The maximum deflection angle of the string & $2^{\circ}$ \\
The maximum deflection angle of the riser & $9^{\circ}$ \\
\hline
\end{tabular}

Table 2. Basic parameters of parallel strings.

\begin{tabular}{lc}
\hline Parameter & Value \\
\hline Length of slip joint & $38.65 \mathrm{~m}$ \\
The weight of slip joint & $407.78 \mathrm{kN}$ \\
Length of riser joint & $16.764 \mathrm{~m}$ \\
The outer diameter of the riser & $0.5334 \mathrm{~m}$ \\
Wall of riser & $0.0222 \mathrm{~m}$ \\
Rotational stiffness of upper flexible joint & $12.9 \mathrm{kN} \mathrm{m} /{ }^{\circ}$ \\
Rotational stiffness of lower flexible joint & $92 \mathrm{kN} \mathrm{m} /{ }^{\circ}$ \\
The outer diameter of the drill pipe & $0.149 \mathrm{~m}$ \\
Wall of drill pipe & $0.0105 \mathrm{~m}$ \\
The height of high-pressure wellhead & $1.75 \mathrm{~m}$ \\
The weight of high-pressure wellhead & $28.73 \mathrm{kN}$ \\
The height of slight-pressure wellhead & $0.99 \mathrm{~m}$ \\
The weight of slight-pressure wellhead & $13.17 \mathrm{kN}$ \\
Length of LMRP and BOP & $17.82 \mathrm{~m}$ \\
The weight of LMRP and BOP & $1470 \mathrm{kN}$ \\
Length of conductor & $83 \mathrm{~m}$ \\
The outer diameter of the conductor & $0.9144 \mathrm{~m}$ \\
Wall of conductor & $0.0381 \mathrm{~m}$ \\
Elastic Modulus of steel & $207 \mathrm{GPa}$ \\
Poisson ratio & 0.3 \\
\hline
\end{tabular}

LMRP: Lower Marine Riser Package; BOP: Blowout Preventer.

Figure 7. The upstream riser A (B) corresponding to downstream string A (B), they are a pair of parallel strings. Figure $7 \mathrm{a}$ indicates that the upstream riser has a spline shape, and the displacement of the riser increases with depth under the currents. Another significant observation from the tests is that the risers are kept in mechanical contact after crashing due to changes in the force field (DNVRP-F203, 2009). The collision force at the bottom between two strings is $5.63 \mathrm{kN}$, which will cause damage to the strength of parallel strings. The reason for this phenomenon is that the bottom of the downstream string is connected to the subsea wellhead. Therefore, from this point of view, effective measures should be taken to decrease the deformation of upstream riser. Regarding the downstream string, the displacement generally increases firstly and then decreases with water depth. The maximum displacement of the downstream string is $6.2 \mathrm{~m}$, which occurs at a depth of water $846 \mathrm{~m}$. The maximum displacement of the down- stream string reduces by half when the shielding effect is not considered. This phenomenon validates the hydrodynamic theory very well taking into account the wake shielding effects. The interference results can be identified clearly by the lateral displacement.

Strength analysis results of parallel strings indicate that the equivalent stress, bending moment much smaller than the limiting criteria except for deflection angle, as shown in Figure 7b. We can conclude that the rotation of the upstream riser tends to decrease gradually with water depth from top to bottom. The top of the riser has a maximum angle of $2.2^{\circ}$ due to the constraint of the tensioner and the influence of the splash zone, which is still less than the permission angle of the riser of $9^{\circ}$. The angle of the downstream string first increases and then decreases with water depth in general and changes suddenly at the junction of the pipe string and the wellhead. The reason for this is that the wellhead constraint can provide bigger resistance forces to restrict the string rotation. When the shielding effect is not considered the rotation angle is greater than $2^{\circ}$, which exceeds the limiting criteria for drilling operations. However, the rotation angle is less than $2^{\circ}$ when it is considered. Although the wake shielding effect can reduce the displacement of the downstream string increasing the risk of parallel string interference, on the contrary, it can also control the rotation angle of the downstream string to reduce the risk of structural strength failure of the parallel string.

\subsubsection{The analysis of influence factors}

The collision of parallel strings is influenced by many factors such as sea conditions, platform offset, and string configuration. These operational factors and environmental factors could affect single riser deformation during riser installation (Wang et al., 2015). Hence, in order to further studies on the interference law of parallel strings, the influencing factors of the current, the platform offset, the weight of the BOP and the wall thickness of the upstream riser are analyzed in this paper, and the results are shown in Figure 8.

The results of the comparative analysis are shown in Figure $8 \mathrm{a}$ and the surface currents are selected from $0.5 \mathrm{~m} / \mathrm{s}, 0.6 \mathrm{~m} / \mathrm{s}$ and $0.7 \mathrm{~m} / \mathrm{s}$, respectively. With the increase of surface current velocity, the lateral displacement of the parallel strings increased significantly, and the displacement at the bottom of the upstream riser increased from $9.58 \mathrm{~m}$ to $15.47 \mathrm{~m}$ with the parallel strings collide seriously. The influencing results of platform offset are shown 


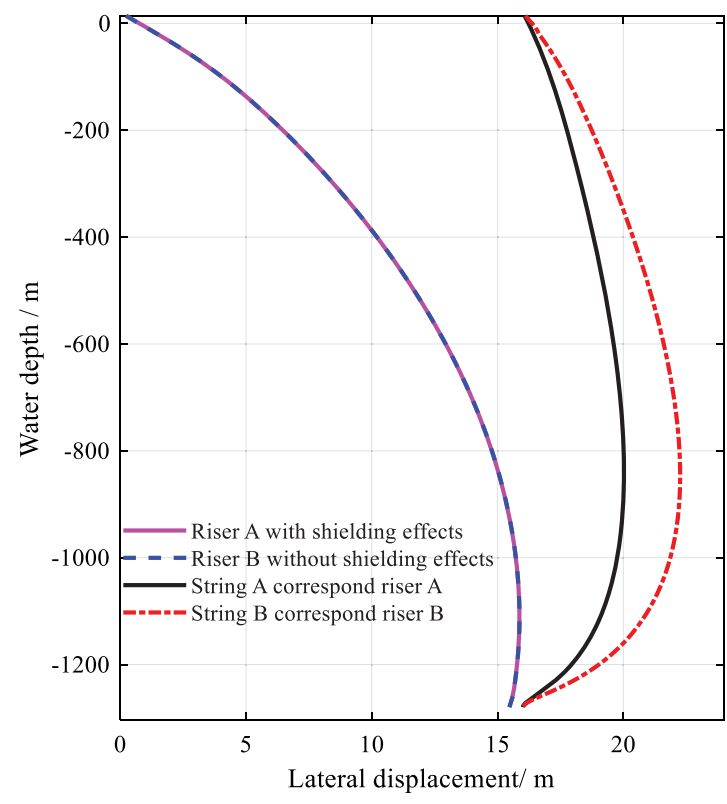

(a)

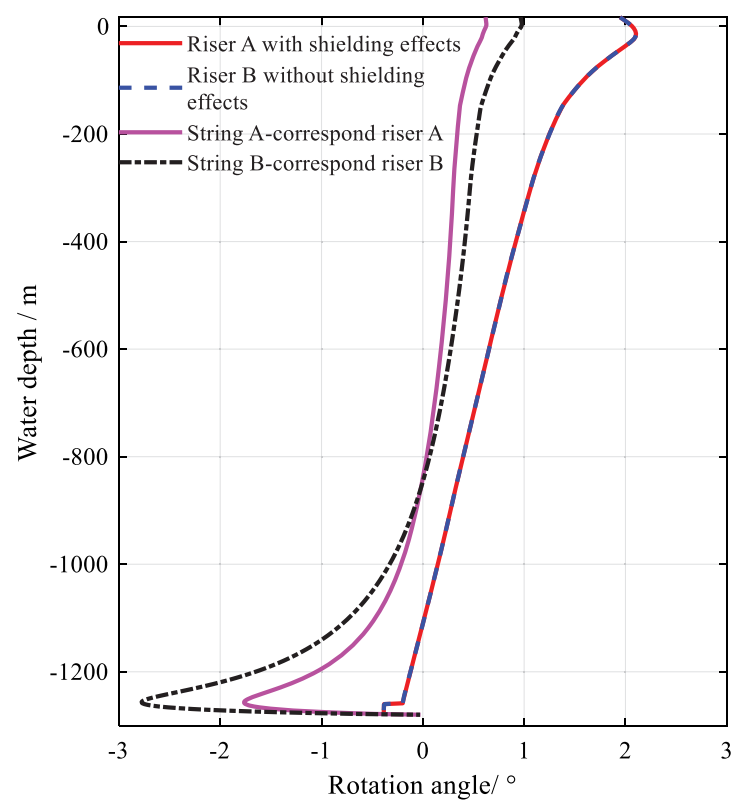

(b)

Fig. 7. Interference analysis results of parallel strings: (a) Displacement of parallel strings, and (b) rotation of parallel strings.

in Figure 8b. The surface current is $0.6 \mathrm{~m} / \mathrm{s}$, and the platform offsets are selected from $-5 \mathrm{~m}$ to $5 \mathrm{~m}$. The deformation of the upstream risers is consistent under different platform offsets and shift to downstream with the platform offset until impacts on downstream string. The top displacement of the downstream string varies with the platform offset, and the bottom connected to the wellhead does not change. The reason for this phenomenon is that the deformation of riser is dominated by current rather than platform offset.

The surface velocity is $0.6 \mathrm{~m} / \mathrm{s}$, and the interference analysis results for different lowering weights of BOP are shown in Figure 8c. The analysis results show that the weight of BOP mainly affects the displacement of the upstream risers. The bottom displacement of the upstream riser will increase by $1.2 \mathrm{~m}$ for each additional $50 \mathrm{kN}$, which has little effect on the offset of the downstream string. From this perspective, BOP with heavier gravity could be a better choice. Figure 8d indicates the displacement results under different riser wall thicknesses. The surface current is $0.6 \mathrm{~m} / \mathrm{s}$, and the wall thickness of the riser is analyzed by three sizes of $0.9375 \mathrm{in} ., 0.875 \mathrm{in}$. and 0.625 in., which are commonly used on the platform. Similar to the influence of the BOP weights, the bigger the riser wall thickness is, the smaller the lateral distance between parallel strings. The reason for this is that thicker risers can provide bigger gravity to compete with lateral combined action generated by wave and current.

\subsection{Design of operability envelopes for parallel strings}

According to the interference analysis of influence factors for the parallel strings, the surface current velocity and platform offset are the main influence factors. However, the operational process and interference limiting criteria of parallel strings are complicated. To avoid the interference collision of parallel strings, it is necessary to determine the safety operation areas of parallel string. The BOP weights and riser thicknesses are important design consideration for designers before the equipment were manufactured. However, the platform offset and surface current can be monitored and controlled by field workers. Hence, considering the platform offset and surface current synthetically, the operability envelopes are designed based on the limiting criteria and lowering process of the upstream riser for the dual derrick drilling operation.

Based on the developed methodology, the operability envelopes for the upstream riser lowering depths at $330 \mathrm{~m}, 660 \mathrm{~m}, 990 \mathrm{~m}$, and $1300 \mathrm{~m}$ are determined respectively, and the analysis results are shown in Figure 9. The envelopes are classified into red, orange, yellow and green four areas according to the limiting of parallel strings. The red area indicates that both string strength criteria and the interference limits are exceeded simultaneously, which means that the parallel string will fracture or interfere with the collision. The yellow area indicates that the parallel strings will break, wear or failure; the orange area indicates that the parallel string will collide. Moreover, the green area indicates that the parallel strings can perform drilling operation normally.

The operability envelope of upstream riser lowering depth at $330 \mathrm{~m}$ is shown in Figure 9a, which can be seen the safe working area of the parallel string is tapered. The platform offset is gradually reduced with the surface current velocity increasing. The maximum platform offset allowed for operation of parallel strings is $55 \mathrm{~m}$, and the maximum surface velocity is $1.4 \mathrm{~m} / \mathrm{s}$. Interference may occur when the maximum platform offset reaches $175 \mathrm{~m}$ as the lowering 


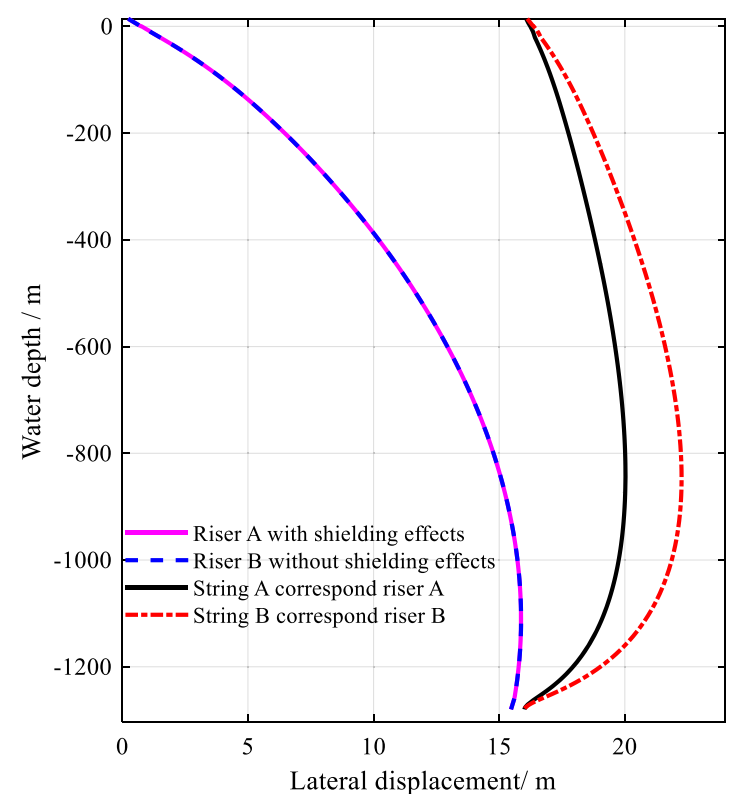

(a)

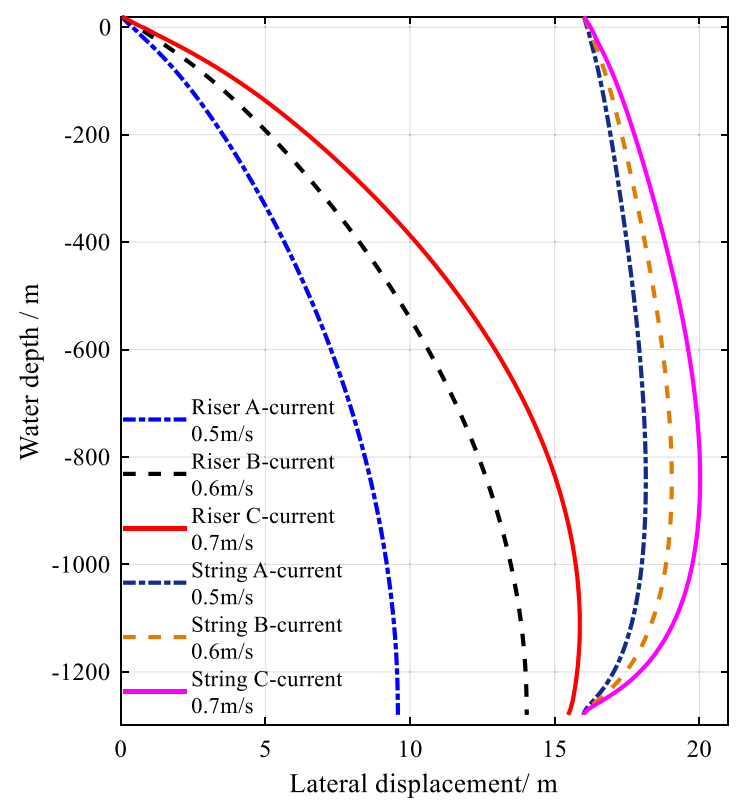

(c)

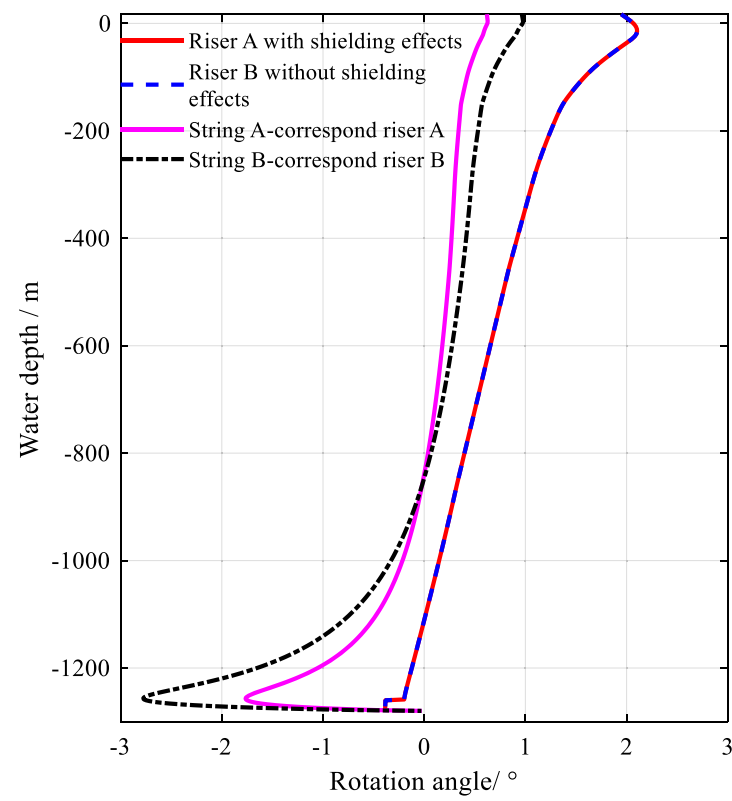

(b)

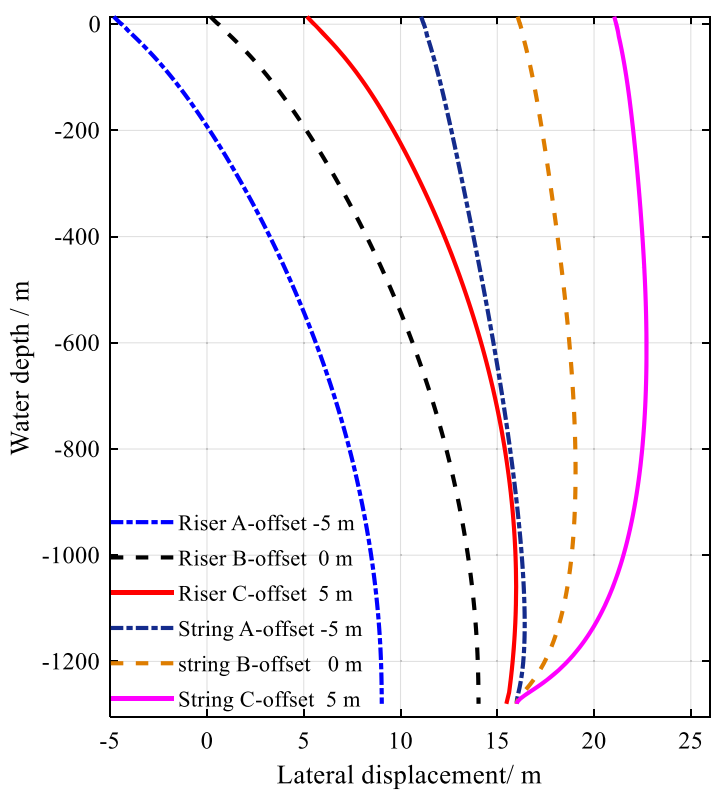

(d)

Fig. 8. Analysis results of influencing factors for parallel strings: (a) Different surface currents, (b) different platform offsets, (c) different BOP weights, and (d) different riser thicknesses.

depth of riser is shallow. Hence, the limiting factors in the early stage of lowering upstream riser are the string strength criteria. It can be seen from Figure $9 \mathrm{~b}$ that when the lowering depth of the upstream riser is $660 \mathrm{~m}$, the displacement of the BOP will increase significantly due to the increasing length of the riser, and the corresponding interference area of the parallel strings will be significantly reduced. Under this condition, the operability envelope is jointly determined by the string strength criteria and the interference limits. When the surface current is greater than
$0.6 \mathrm{~m} / \mathrm{s}$, the envelope is entirely restricted by the interference collision limits. Nevertheless, a small string damage area of the envelope is limited by the strength criteria when the surface current is less than $0.6 \mathrm{~m} / \mathrm{s}$.

The operability envelope is shown in Figure $9 \mathrm{c}$ when the upstream riser is lowering to a depth of $990 \mathrm{~m}$. The interference criteria of parallel strings completely determine the envelope. The BOP displacement will gradually increase with the increasing length of lowering riser, and the area of the string interference has narrowed. Since the distance 


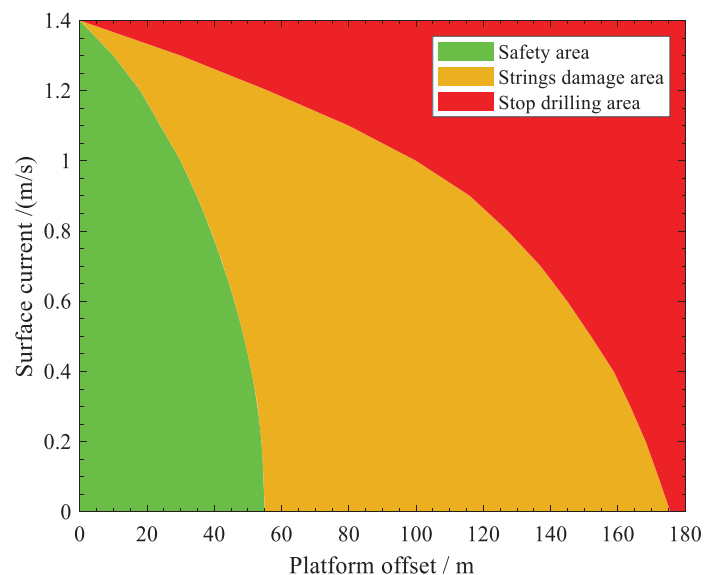

(a)

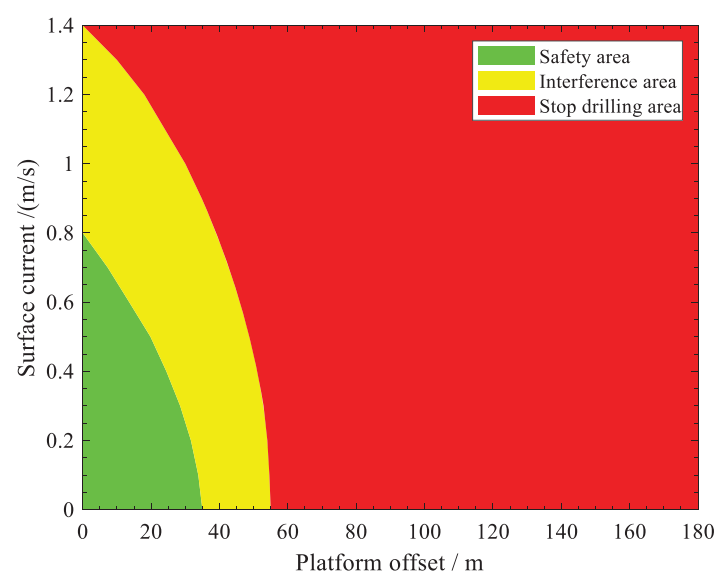

(c)

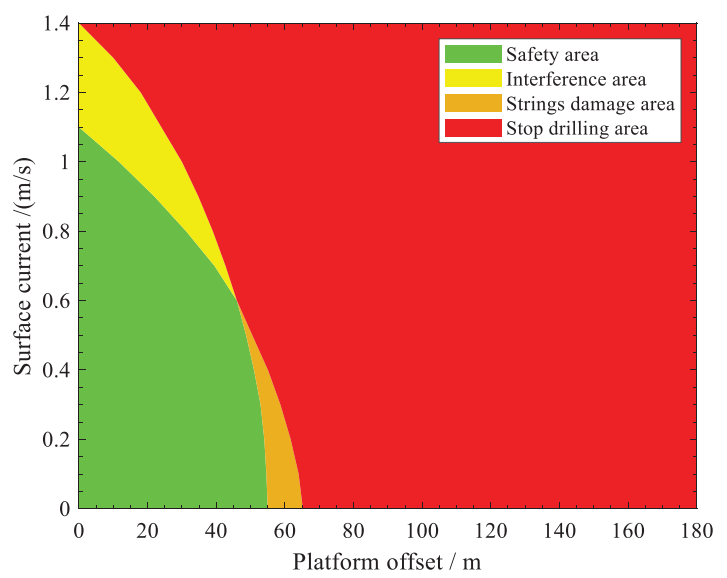

(b)

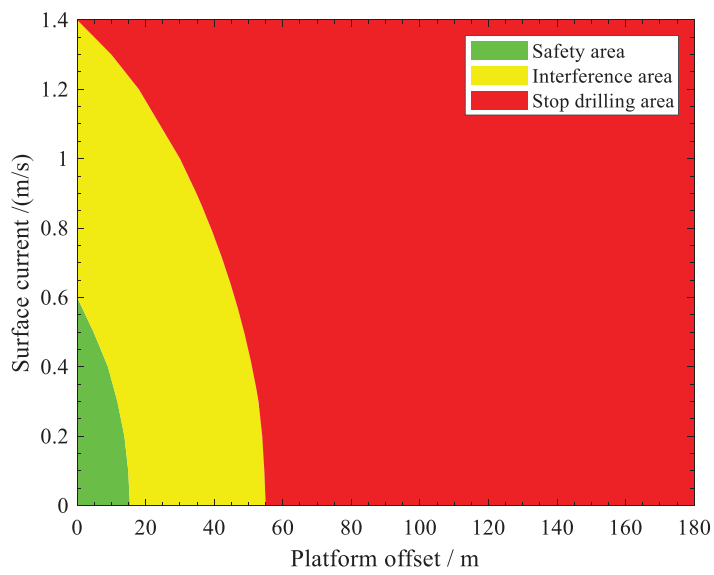

(d)

Fig. 9. Operability envelopes for parallel strings under different lowering depths: (a) Lowering depth at $330 \mathrm{~m}$, (b) lowering depth at $660 \mathrm{~m}$, (c) lowering depth at $990 \mathrm{~m}$, and (d) lowering depth at $1300 \mathrm{~m}$.

between two strings is limited to $16 \mathrm{~m}$, when the surface current reaches $0.8 \mathrm{~m} / \mathrm{s}$, the BOP connected to the end of the riser will collide with the downstream string even platform non-offset in this case. Similarly, the maximum allowable platform offset is $35 \mathrm{~m}$. When the riser run to the seabed completely the envelope is shown in Figure 9d, suspending from the platform ready for installation. The safe area of the parallel string is wholly determined by the interference collision criteria. At this time, in order to ensure the safety of the working string, the current velocity of the sea should not exceed $0.6 \mathrm{~m} / \mathrm{s}$ and the platform without offset. Otherwise, the strings will collide. Therefore, it is necessary to maintain the stability of the platform according to the field sea conditions.

\section{Conclusion}

This study addresses a challenging problem in the engineering of parallel strings for deepwater dual derrick operation, that is, the interference analysis for parallel strings due to the shielding effect of current, the complex operations and environment during operation of dual derrick platform. A mechanical model for deepwater parallel strings is established to analyze the response of parallel strings. The research results of the parallel strings system validate the effectiveness of theoretical analysis very well considering the hydrodynamic wake shielding effects. The results prove that the collision will occur during the operation, the collision force at the bottom of the two strings can reach $5.63 \mathrm{kN}$, which will cause serious damage to the strength of parallel strings. Furthermore, the influence factors of platform offsets, surface currents, BOP weights and wall thicknesses of the upstream riser on the interference analysis of parallel strings are analyzed. It can be concluded that the platform offset and surface currents are the significant influence factors.

An original efficient methodology of operability envelopes for parallel strings is presented to control the occurrence of interference accidents. The interference limiting factors of parallel strings and operational limiting criteria can be considered. Thus, the operability envelopes are developed by the permissible platform offsets and surface currents for different depths of the upstream riser. The 
safety area of operability envelopes is continuously decreasing with the increasing length of the upstream riser, which can provide an operation reference for drillers. The proposed methodology of operability envelopes is more applicable to field practice. Additionally, to avoid interference accidents effectively other measurements can be incorporated into the operational procedures, e.g., observing present sea current and monitoring the distance between the two strings in the water.

In the present study, to analyze the response of parallel strings, a mechanical model is established considering hydrodynamic effects, and a novel method of operability envelopes is proposed to avoid the accident of interference collisions. Additionally, further studies are required to improve the design method and determine the operability envelopes considering the dynamic response of the platform during the lowering operation of the parallel strings.

Acknowledgments. This work was supported by the China National Key Research and Development Program (Grant numbers: 2016YFC0304005, 2017YFC0804501), the Fundamental Research Funds for Innovation Program of Seventh-generation Ultra Deepwater Drilling Platform [grant numbers 2016[24]], and the Fundamental Research Funds for the Central Universities (18CX02157A).

\section{References}

Blevins R.D. (1994) Flow induced vibrations, Krieger Publishing Company, Malabar.

Campbell M., Kaculi J., Koska R., Mills D. (2013) Maximizing drilling riser installation operability for TLP top tensioned risers, in: Proceedings of the ASME 2013 32nd International Conference on Ocean, Offshore and Arctic Engineering, ASME, Nantes, France.

Chang Y., Song Q., Zhang H., Zhang W. (2017) Collision analysis of risers in tandem for TLP under typhoon, J. Ship Mechanics, 21, 5, 633-641.

Det Norske Veritas. (2009) Offshore standard DNV-RP-F203: Riser interference, Hovik, Norway.

Det Norske Veritas. (2010) Offshore standard DNV-OS-F201: Dynamic risers, Hovik, Norway.

Do K.D. (2018) Boundary control of transverse motion of flexible marine risers under stochastic loads, Ocean Eng., 155, $156-172$.

Dong X., Cao S., Tang H., Chen J. (2011) Ocean drilling manual, Petroleum Industry Press, Beijing.

Drumond G.P., Pasqualino I.P., Pinheiro B.C., Estefen S.F. (2018) Pipelines, risers and umbilicals failures: A literature review, Ocean Eng., 148, 412-425.

Fontaine E., Capul J., Rippol T., Lespinasse P. (2013) Experimental and numerical study of wake interference and clashing between steel catenary risers, J. Offshore Mech. Arct. Eng., 135, 1, 011701.

Fu P., Leira B.J., Myrhaug D. (2017) Reliability analysis of wake-induced collision of flexible risers, Appl. Ocean Res. 62, 49-56.

Fu P., Leira B.J., Myrhaug D. (2018) The effect of local wake model on collision probability for risers subjected to current and random waves, Ocean Eng. 162, 331-340.
Hall J.E., Herrmann R.P., Holt C.A., Straub W.J., Weber R.G. (1999) Use of the dual-activity drillship as a field development tool, in: Offshore Technology Conference, OTC 10891, Houston, TX.

He J.W., Low Y.M. (2012) An approach for estimating the probability of collision between marine risers, Appl. Ocean Res., 35, 68-76.

He J.W., Low Y.M. (2014) A collision-allowed approach for probabilistic assessment of riser interference, Ocean Eng., 76, 21-31.

Huang S. (2010) Instability of a vertical riser in the wake of an upstream vertical riser, Appl. Ocean Res., 32, 3, 351-357.

Huera-Huarte F.J., Bearman P.W. (2011) Vortex and wakeinduced vibrations of a tandem arrangement of two flexible circular cylinders with near wake interference, J. Fluids Struct. 27, 2, 193-211.

Huera-Huarte F.J., Gharib M. (2011) Vortex and wake-induced vibrations of a tandem arrangement of two flexible circular cylinders with far wake interference, J. Fluids Struct., 27, 5-6, 824-828.

Huse E., Kleiven G. (2000) Impulse and energy in deepsea riser collisions owing to wake interference, in: Offshore Technology Conference, OTC 11993, Houston, TX.

Huse E. (1993) Interaction in deep-sea riser arrays, in: Offshore Technology Conference, OTC7237, Houston, TX.

Huse E. (1996) Experimental investigation of deep sea riser interaction, in: Offshore Technology Conference, OTC8070, Houston, TX.

Ju S., Chang Y., Chen G., Liu X., Xu L., Wang R. (2012) Envelopes for connected operation of the deepwater drilling riser, Petrol. Explor. Dev., 39, 1, 113-118.

Kalleklev A.J., Mørk K.J., Sødahl N., Nygård M.K., Horn A.M. (2003) Design guideline for riser collision, in: Offshore Technology Conference, OTC 15383, Houston, TX.

Liu D., Wang Y., Teng Y., He G., Qu Y., Chen Y., Chen M. (2017) Optimal selection and adaptable transformation of marine NGH trial production platform in the South China Sea, Ship Building of China, 58, A01, 320-329.

Liu K., Chen G., Zhu G., Zhu J. (2019) Nonlinear self-induced vibration and operability envelope analysis of production strings in marine natural gas development, Int. J. Nav. Arch. Ocean Eng. 11, 1, 344-352.

Liu X., Chen G., Chang Y., Liu K., Zhang L., Xu L. (2013) Analyses and countermeasures of deepwater drilling riser grounding accidents under typhoon conditions, Petrol. Explor. Dev. 40, 6, 791-795.

Munch-Søegaard L., Nergaard A. (2001) Offshore drilling experience with dual derrick operations. in: $S P E / I A D C$ Drilling Conference, SPE/IADC 67706, Amsterdam.

Nygård M.K., Botros F.R., Hannus H. (2001) Evaluation of effect of contact between top tensioned risers in deep and ultra deep waters, Proceedings of the 11th International Offshore and Polar Engineering Conference, ISOPE, Stavanger, Norway.

Rustad A.M., Larsen C.M., Sorensen A.J. (2008) FEM modelling and automatic control for collision prevention of top tensioned risers, Mar. Strut., 21, 1, 80-112.

Rustad A.M., Kalleklev A.J., Sdahl N. (2009) Recommended Practice on Riser Interference, in: Offshore Technology Conference, OTC 20066, Houston, TX.

Sagatun S.I., Herfjord K., Holmås T. (2002) Dynamic simulation of marine risers moving relative to each other due to vortex and wake effects, J. Fluids Struct., 16, 3, 375-390.

Sumner D. (2010) Two circular cylinders in cross-flow: a review, J. Fluids Struct., 26, 6, 849-899. 
Wang Y., Gao D., Fang J. (2015) Mechanical behavior analysis for the determination of riser installation window in offshore drilling, J. Nat. Gas Sci. Eng., 24, 317-323.

Wilburn M.D., Steddum R.E., Shaughnessy J.M. (1998) Deepwater rig design for 10,000 feet of water, in: Offshore Technology Conference, OTC 8753, Houston, TX.

Wu W., Huang S., Barltrop N. (2003) Multiple stable/unstable equilibria of a cylinder in the wake of an upstream cylinder, $J$. Offshore Mech. Arct. Eng., 125, 2, 103-107.

Yuan Y., Xue H., Tang W. (2018) Numerical analysis of VortexInduced Vibration for flexible risers under steady and oscillatory flows, Ocean Eng., 148, 548-562.
Yue J., Qi Y., Xiao W., Yang L., Chen J. (2009) Rig mode option for semi-submersible drilling platform, Petrol. Explor. Dev. 36, 6, 776-783.

Zdravkovich M.M. (1997) Flow around circular cylinders, vol. 1: Fundamentals, Oxford University Press, New York.

Zdravkovich M.M. (2003) Flow around circular cylinders, vol. 2: applications, Oxford University Press, New York.

Zhang M., Fu S., Song L., Tang X., He Y. (2018) A time domain prediction method for the vortex-induced vibrations of a flexible riser, Mar. Strut., 59, 458-481. 